\title{
Standardization of Agrobacterium mediated genetic transformation in Indica rice cV BPT-5204
}

Mahendranath Gandikota ${ }^{1,2,3}$ Krishnakanth Yadav T. ${ }^{1}$ Jagadish P. ${ }^{1}$ Raghurami Reddy M. ${ }^{2,3}$, Balachandran S.M. ${ }^{2}$, Siddiq E.A. ${ }^{1}$, Yamini K.N. ${ }^{1}$, Anuradha G. ${ }^{*}$

${ }^{1}$ Institute of Biotechnology, Professor Jayashankar Telangana State Agricultural University (PJTSAU), Rajendranagar, Hyderabad, India.

2ICAR-Indian Institute of Rice Research, Rajendranagar, Hyderabad, India.

${ }^{3}$ Department of Genetics and Biotechnology, Osmania University, Hyderabad, India.

Received: 11/22/2017; Revised: 1/15/2018; Accepted: 1/28/2018

\begin{abstract}
The BPT-5204 genotype from Indica rice cultivar (cv) is recalcitrant and showed low transformation frequency compare to japonica rice $c v$. Here we have optimized the efficient transformation protocol to minimize the time scale and enhance the transformation frequency by altering the key parameters like, acetosyringone (AS) concentration, optical density of bacterial culture and co-cultivation time. Highly proliferated 21 days old Scutellum derived embryogenic calli were infected with Agrobacterium tumefaciens harbouring pCAMBIA2300-Ds-En-Bar binary vector. T-DNA contains tetrameric CaMV35S enhancer elements along with bar gene which embedded between the transposable $D s$ element. At $0.5 \mathrm{OD}_{600 \mathrm{~nm}}$ of Agrobacterium culture and co-cultivation for 2 days on MS co-cultivation medium containing $100 \mu \mathrm{M}$ acetosyringone proved to be optimal and attained $6.2 \%$ of transformation efficiency. The transformed calli and regenerated plantlets were proliferated on Murashige and Skoog (MS) medium containing phosphinothricin (PPT). Polymerase chain reaction (PCR) confirmed that intact T-DNA was successfully integrated in the rice genome. This protocol can be employed to develop transgenic rice plants with gain of functional mutagenesis.
\end{abstract}

Keywords: Agrobacterium tumefaciens; BPT-5204; Embryogenic calli; Activation tagging

\section{Introduction}

Rice (Oryza sativa L.) is one of the most important cereal crop and a primary food source for half of the world's population. The increasing demand for rice provide chances for biotechnologists to develop efficient, quick and reproducible transformation protocols to meet the demand. Major constraints in rice production are biotic and abiotic stress causing significant yield losses up to 10 and $30 \%$ in rice growing countries (Krishnaiah and Varma 2012). Genetic engineering is alternative to overcome these problems associated with narrow genetic base and tissue culture is the pre requisite event for generation of transgenic plants. More sustained production of rice is essential which in turn requires an efficient protocol for its regeneration and transformation. Various gene transfer protocols were developed for rice among them Agrobacterium tumefaciens genetic transformation is a preferred method due to stable and transfer of larger DNA segments and low integration copy number of transfer-DNA (T-DNA) into the plant genome (Gelvin 2000). Different types of explants are used for transformation, such as mature seed derived calli, immature embryo-derived calli, leaf base derived calli and shoot apex. Among these explants, calli derived from mature seeds showed high transformation efficiency (Hiei and Komari 2008; Rance et al., 1994; Manimaran et al., 2013). In rice, many factors are influencing the efficiency of

\section{*Corresponding Author:}

Prof. Anuradha G., Ph.D.

Institute of Biotechnology (IBT)

Professor Jayashankar Telangana State Agricultural University (PJTSAU),

Rajendranagar, Hyderabad, India.

E-mail: saps_61@yahoo.com
T-DNA delivery to plant cell such as type of explants, optical density of Agrobacterium, infection time, co-cultivation medium with acetosyringone for vir gene induction. An excessive number of bacteria can stress plant cells and affect their regeneration potential, whereas low concentration can reduce the frequency of T-DNA transfer (Fitch et al., 2011; Zhao et al., 2010). In general, cocultivation time of 2-3 days is standard for most transformation systems (Mourgues et al., 1996; Cervera et al., 1998). Phenolic compounds like acetosyringone are recommended for enhancing plant genetic transformation (Kumlehn et al., 2006; Kavitha et al., 2010). Rice became a model crop for genetic and functional genomic studies among monocots (Jung et al., 2008). In recent years, considerable progress has been made in the improvement of important agronomic traits of rice through biotechnological approaches (Hao et al., 2009; Skamnioti and Gurr 2009). Insertional mutagenesis is one of the most useful methods for analyzing gene function (Upadhyaya et al., 2011). Activation tagging has become an important tool in targeted improvement and gene function studies of indica rice (Manimaran et al., 2017). Hence, the present investigation was undertaken with the objective of developing an efficient Agrobacteriummediated genetic transformation method and subsequent transgenic plant recovery system using 
embryogenic calli of Indica rice $c v$ BPT-5204. Various factors influencing the transformation frequency such as optical density (OD), cocultivation duration and acetosyringone concentration were evaluated.

\section{Materials and Methods}

\section{Plant material and methods}

Embryogenic calli derived from mature rice seeds of Oryza sativa L. subsp. Indica cv BPT-5204. Surface sterilization of dehusked seeds with autoclaved double distilled water for four times for $1 \mathrm{~min}$ each followed by ethanol (70\%) for $2 \mathrm{~min}$, mercuric chloride $(0.1 \%)$ for $3-4 \mathrm{~min}$ and then washed with double distilled water for three times. These seeds were dried on sterile filter paper.

\section{Agrobacterium strain and plasmid construct} Agrobacterium tumefaciens EHA105 harbouring the binary vector pCAMBIA2300-Ds-En-Bar. The TDNA region contains the 4XCaMV35S enhancer elements transcriptional activation of flanking plant genes, Bar gene under control of CaMV35S promoter were embedded in between transposable element $D s 5^{\prime}$ to $3^{\prime}$ (Fig. 1). Culture was maintained in glycerol stocks at $-80^{\circ} \mathrm{C}$.
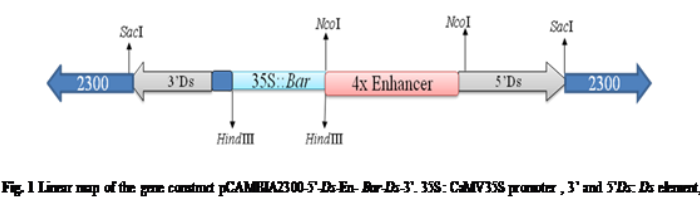

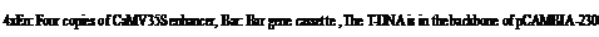

Agrobacterium mediated genetic transformation

Surface sterilized seeds were inoculated on to the callus induction (CI) medium (Fig 2 A) [Murashige and skoog (MS) salts supplemented with 2,4-D 2.5 $\mathrm{mg} / \mathrm{L}$, kinetin $0.5 \mathrm{mg} / \mathrm{L}$, proline $500 \mathrm{mg} / \mathrm{L}$, casein enzymatic Hydrolysate $500 \mathrm{mg} / \mathrm{L}$ and maltose 30 $\mathrm{g} / \mathrm{L}, \mathrm{p}^{\mathrm{H}}$ of the medium was adjusted to 5.8] solidified with $0.4 \%$ phytagel and autoclaved at $121^{\circ} \mathrm{C}$ for $15 \mathrm{~min}$ at $15 \mathrm{psi}$ pressure. The calli derived from Scutellum region of the rice seeds were subculture and incubated on fresh CI medium for 5 days (Fig. 2B).

Preparation of primary Agrobacterium culture Agrobacterium culture was prepared by inoculating single colony from a freshly streaked plate, in a 250 $\mathrm{ml}$ baffled conical flask containing $50 \mathrm{ml}$ of autoclaved liquid YEB medium $(5 \mathrm{~g} / \mathrm{L}$ Beaf extract, $1 \mathrm{~g} / \mathrm{L}$ yeast extract, $5 \mathrm{~g} / \mathrm{L}$ peptone and $5 \mathrm{~g} / \mathrm{L}$ sucrose and $1.5 \%$ agar, $\left.\mathrm{p}^{\mathrm{H}} 7.0\right)$ supplemented with rifampicin $10 \mathrm{mg} / \mathrm{L}$, kanamycin $50 \mathrm{mg} / \mathrm{L}$. The culture was incubated at $220 \mathrm{rpm}$ in dark at $28^{\circ} \mathrm{C}$ varying optical density (OD ${ }_{600 \mathrm{~nm}}$ ) between 0.1 to 0.7 and these Agrobacterium cells were pelleted by centrifugation at $3100 \mathrm{rpm}$ for $15 \mathrm{~min}$ at $28^{\circ} \mathrm{C}$.
Agrobacterium infection and selection of transformed calli

The harvested Agrobacterium cells were resuspended in MS resuspension liquid (MSL) medium containing 50 to $200 \mu \mathrm{M}$ of acetosyringone (sigmaAldrich, St Louis, USA) (MS basal salts, $68 \mathrm{~g} / \mathrm{L}$ sucrose, $36 \mathrm{~g} / \mathrm{L}$ glucose, $100 \mathrm{mg} / \mathrm{L}$ myo-inositol, $876 \mathrm{mg} / \mathrm{L}$ L-glutamin, $3 \mathrm{~g} / \mathrm{L} \mathrm{KCl}, 4 \mathrm{~g} / \mathrm{L} \mathrm{MgCl}_{2}$, pH 5.2) and incubated for $28^{\circ} \mathrm{C}$ for $1 \mathrm{hr}$. Scutellum derived embryogenic calli (Fig. 2 C) were infected with these various concentration $(0.1$ to 0.7 of $\left.\mathrm{OD}_{600 \mathrm{~nm}}\right)$ Agrobacterium culture for $30 \mathrm{~min}$ with intermitten gentle shaking at $100 \mathrm{rpm}$. The infected embryogenic calli were transfer on sterile filter paper for $3 \mathrm{~min}$ to remove excess bacterial culture. Infected calli were transfer on to the co-cultivation (COC) medium (CI medium with containing 10 $\mathrm{mg} / \mathrm{L}$ glucose, 0 to $150 \mu \mathrm{M}$ acetosyringone, $\mathrm{p}^{\mathrm{H}} 5.2$ solidified with $0.4 \%$ phytagel) for 1 to 3 days of cocultivation time at $28^{\circ} \mathrm{C}$ in dark. The co-cultivated calli were washed with sterile liquid medium containing $250 \mathrm{mg} / \mathrm{L}$ cefotaxime to remove Agrobacterium. The washed calli blot dried on filter paper and transferred on to first selection medium (CI medium containing $250 \mathrm{mg} / \mathrm{l}$ cefotaxime and $5 \mathrm{mg} / \mathrm{L}$ phosphinothricin) and incubated for 3 weeks at $28^{\circ} \mathrm{C}$ in dark. The resistance calli were further selected through two rounds of selection cycles for 15 days each (Fig. 2D). Newly proliferated micro calli generated on selection medium were transfer to the regeneration medium (MS basal salts, $2.5 \mathrm{mg} / \mathrm{L}$ kinetin, $0.5 \mathrm{mg} / \mathrm{L}$ naphthyl acetic acid (NAA), $30 \mathrm{mg} / \mathrm{L}$ sucrose, $20 \mathrm{mg} / \mathrm{L} D$-sorbitol and $\mathrm{pH} 5.8$ solidified with $0.4 \%$ phytagel). The cultures were maintained in dark for 1 week then shifted to photo period of 16-hrs light/8 hrs dark for 3 weeks. For roots development, the regenerated shoots buds were transfer to rooting medium (half strength MS basal salts $15 \mathrm{~g} / \mathrm{L}$ sucrose, $\mathrm{p}^{\mathrm{H}} 5.8$ and $0.3 \%$ phytagel) and maintained at $28^{\circ} \mathrm{C}$ in light for two weeks (Fig. $2 \mathrm{E}$ ). Fully rooted plants were transferred to pots and maintained at transgenic glass house (Fig. 2 F).

Molecular analysis of putative transgenic plants Confirm the presence of transgene in putative transformed and non-transformed plantlets through PCR analysis. Genomic DNA from various independent transgenic lines was extracted by the method described by Dellaporta et al., (1983). Following primers pair was used for PCR analysis: En-F (5'-CAAAGGGTAATATCGGGAAACC-3') and Bar-R (5'-CATCAGATCTCGGTGACG-3'). The reaction mixture of $20 \mu \mathrm{l}$ (total volume) containing 50ng good quality template DNA, 1X Taq buffer (with $25 \mathrm{mM} \mathrm{MgCl} 2$ ), $0.125 \mathrm{mM}$ dNTPS, $5 \mathrm{pM}$ primers and $1 \mathrm{U}$ Taq DNA polymerase and the profile was as follows: initial denaturation $95^{\circ} \mathrm{C}$ for 5 min followed by 32 cycles; denaturation $95^{\circ} \mathrm{C}$ for $30 \mathrm{sec}$; annealing $58^{\circ} \mathrm{C}$ for $30 \mathrm{sec}$; elongation $72^{\circ} \mathrm{C}$ for $1 \mathrm{~min}$ with final extension $72^{\circ} \mathrm{C}$ for $5 \mathrm{~min}$. The 
amplified PCR product was resolved on 1.2\% agarose gel electrophoresis.

\section{Result and Discussion}

Modified protocol for callus induction and transformation

Elite Indica rice cultivar BPT-5204 was used for initial standardization of various parameters for enhancement of embryogenic callus induction and regeneration. This optimized protocol was used for further experiments to enhance transformation as well as regeneration efficiency. Maximum callus proliferation was observed after 19-21 days (fig 2 C). The callus induction percentage was found to be $85 \%$. The embryogenic calli formed on the CI medium were creamy white, compact and globular structure. Pre-cultured embryogenic calli (for 5 days) on CI medium (2.5 mg/L 2, 4-D, $0.5 \mathrm{mg} / \mathrm{L}$ kinetin) was used as an explants for transformation.

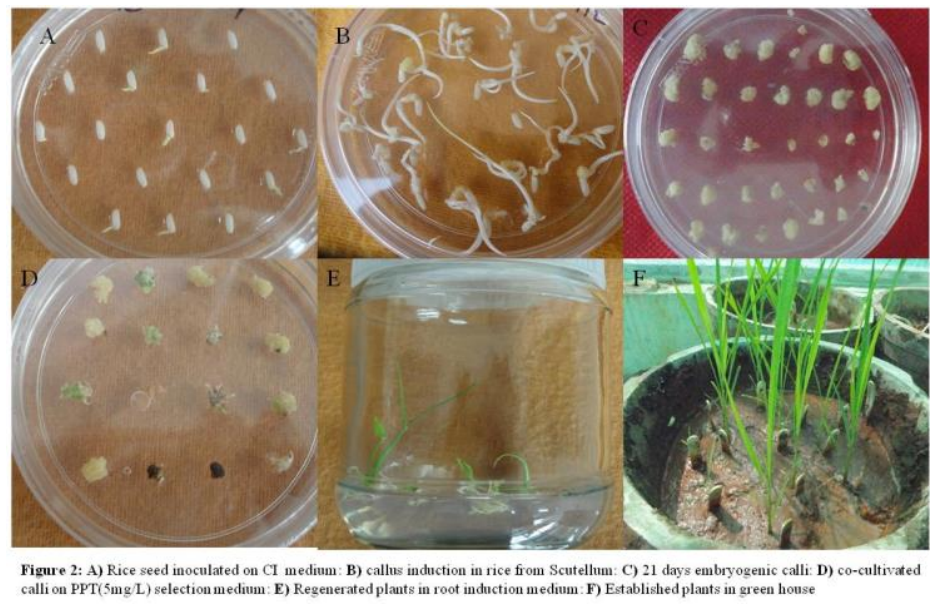

\begin{abstract}
Effect of optical density (OD) transformation frequency
\end{abstract}

Pre-cultured embryogenic calli were co-cultivated with different optical density $\left(\mathrm{OD}_{600 \mathrm{~nm}}\right)$ values $(0.1$, 0.3, 0.5 and 0.7) of Agrobacterium culture with $1 \mathrm{hr}$ of infection time. It was observed that the increase of Agrobacterium OD was directly proportional to increase in the percentage of survival calli. Transformation event was not detected at lower bacterial density (0.1). Embryogenic calli infected with Agrobacterium culture of an $\left(\mathrm{OD}_{600 \mathrm{~nm}}\right)$ of 0.5 were observed to give the best transformation frequency (16\%) (Table 1). However, increasing the Agrobacterium concentration to $\left(\mathrm{OD}_{600 \mathrm{~nm}}\right) 0.7$ which negatively affects the transformation frequency. The $\left(\mathrm{OD}_{600 \mathrm{~nm}}\right)$ of 0.5 value might be representing the active $\log$ phase of Agrobacterium growth and thus very effective for transformation. Previous reports indicated that the $\mathrm{OD}_{600 \mathrm{~nm}}$ of the bacterial density in a range from 0.3 to 1 is suitable for transformation (Aananthi et al., 2010; Baskaran et al., 2012). In the present study, $\mathrm{OD}_{600 \mathrm{~nm}}$ of 0.5 proved to be the best concentration of Agrobacterium for successful transformation in Indica rice cultivar BPT-5204.

Table.1: Influence of concentration of Agrobacterium on transformation frequency

\begin{tabular}{cc}
\hline $\begin{array}{c}\text { Agrobacterium } \\
\text { concentration (OD } \text { (600nm })\end{array}$ & $\begin{array}{c}\text { Phosphinothricin } \\
\text { resistant calli \% }\end{array}$ \\
\hline 0.1 & 0 \\
0.3 & $7 \pm 1.7$ \\
0.5 & $16 \pm 1.9$ \\
0.7 & $6 \pm 1.5$ \\
\hline
\end{tabular}

Pre-cultured embryogenic calli were infected for $1 \mathrm{hr}$ with Agrobacterium strain EHA105 containing pCAMBIA 2300-Ds-En-bar and co-cultivation for 2 days on MS basal medium, $100 \mu \mathrm{M}$ concentration of acetosyringone containing respective Agrobacterium concentration (OD). Transformed calli were transferred to selection medium (phosphinothricin $5 \mathrm{mg} / \mathrm{L}$ ). The mean of three independent experiments ( \pm standard error) are provided.

\section{Influence of Addition of acetosyringone on} transformation frequency

Acetosyringone is a phenolic compound produced during plant cell wounding that induces the transcription of Agrobacterium tumifaciens virulence genes that regulate the processing and transfer of $\mathrm{T}$ DNA from A.tumifaciens to plant cells (Shimoda et al., 1990; Gelvin 2003; Tripathi et al., 2010). Higher transformation frequency is achieved by addition of acetosyringone in co-cultivation medium. In present study, embryogenic calli were co-cultivated with Agrobacterium cultures (EHA105: pCAMBIA2300Ds-En-Bar strain) which supplemented with different concentrations of acetosyringone (AS) $(0$, $50,100$ and $150 \mu \mathrm{M})$ to identify the optimized concentration (Table 2). Embryogenic calli infected with Agrobacterium OD ${ }_{600 \mathrm{~nm}}$ of 0.5 and with addition of $100 \mu \mathrm{M}$ acetosyringone showed better response (regenerated calli after two sub-cultures on phosphinothricin selection media) of $18.7 \%$ when compared with other concentrations. 
Table 2: Influence of concentration of acetosyringone transformation frequency

\begin{tabular}{cc}
\hline $\begin{array}{c}\text { Acetosyringone } \\
\text { concentration }\end{array}$ & $\begin{array}{c}\text { Phosphinothricin } \\
\text { resistant calli \% }\end{array}$ \\
\hline $0 \mu \mathrm{M}$ & $5 \pm 0.6$ \\
$50 \mu \mathrm{M}$ & $9 \pm 0.5$ \\
$100 \mu \mathrm{M}$ & $18 \pm 0.7$ \\
$150 \mu \mathrm{M}$ & $7 \pm 0.8$ \\
\hline
\end{tabular}

Pre-cultured embryogenic calli were infected for 30 min with Agrobacterium strain EHA 105 containing pCAMBIA 2300-Ds-En-bar $\mathrm{OD}_{600}$ of 0.5 and cocultivation for 2 days on MS basal medium containing the respective concentration of acetosyringone. Transformed calli were transferred to selection medium (phosphinothricin $5 \mathrm{mg} / \mathrm{L}$ ). The mean of three independent experiments ( \pm standard error) are provided.

Influence of co-cultivation time on transformation frequency

Transformation frequency mainly depends on the duration of co-cultivation of the infected explants with Agrobacterium. The co-cultivation time duration varied with type of explants, genotype of the plant, Agrobacterium strain. Different co-cultivation periods $(0,1,2$ and 3 days) were investigated. The infected calli without co-cultivation period not show any transformation frequency (Table 3). One day cocultivation period gives $10 \%$ transformation frequency, two days co-cultivation periods showed the highest percentage of transformation frequency by $19 \%$ whereas the transformation frequency was decreased dramatically in the case of using three days as a co-cultivation period ( $8 \%$ ). Therefore, two day co cultivation period was considered as optimized and further prolonged exposure of cells (3days) to Agrobacterium may adversely affect the calli which causes the cell death or overgrowth of bacteria. Previous reports indicated embryogenic calli infected with Agrobacterium with two to three days of co-cultivation period resulted in the highest transformation frequency without overgrowth of Agrobacterium as longer time will cause bacterial overgrowth (Rashid et al., 1996; Urushibara et al., 2001; Zhang et al., 2015).

Table 3: Influence of co-cultivation time (days) on transformation frequency

\begin{tabular}{cc}
\hline $\begin{array}{c}\text { co-cultivation time } \\
\text { (days) }\end{array}$ & $\begin{array}{c}\text { Phosphinothricin } \\
\text { resistant calli \% }\end{array}$ \\
\hline 0 & 0 \\
1 & $10 \pm 1.4$ \\
2 & $19 \pm 2.1$ \\
3 & $8 \pm 0.8$ \\
\hline
\end{tabular}

Pre-cultured embryogenic calli were infected for $1 \mathrm{hr}$ with Agrobacterium strain EHA105 containing pCAMBIA 2300-Ds-En-bar $\mathrm{OD}_{600 \mathrm{~nm}}$ of 0.5, MS basal medium containing the $100 \mu \mathrm{M}$ concentration of acetosyringone and respective co-cultivation time (days). Transformed calli were inoculated on selection medium (phosphinothricin $5 \mathrm{mg} / \mathrm{L}$ ). The mean of three independent experiments $(\underline{ \pm}$ standard error) are provided.

\section{Molecular confirmation of the transgenic plants of regenerated plants}

The developed plants were analyzed for transgene through molecular technique. PCR is a sensitive technique allowing genes to be amplified and extracted out of a complex mixture of genomic sequences. Amplified DNA is visualized as distinct bands on agarose gels. Therefore, PCR was utilized to confirm the integration of T-DNA fragments in plantlets. The primers used for PCR were designed to amplify a 900bp fragment of bar gene. Expected fragment was observed in the $27 \mathrm{~T}_{0}$ plantlets. Non transformed control BPT-5204 plantlets did not show any amplification with gene specific primers.

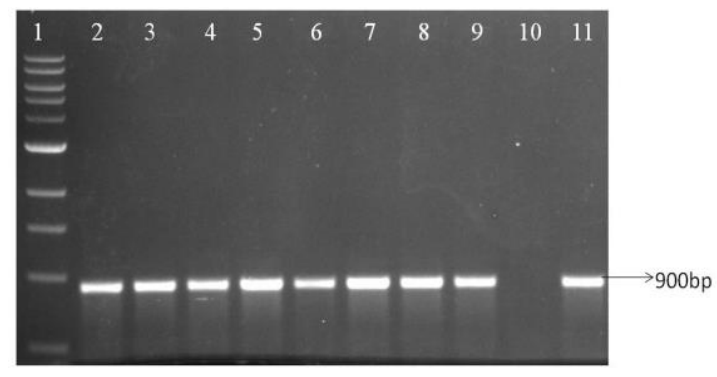

Figure 3 PCR verification of T0 putative trangsenics in rice variety BPT-5204 usimg Bat
primers. Lame 1: 1kb MW marker, 2-9: Putative transgenic plants, 10 : Negative Control, 11: primers. Lame
Positive Control

We have developed a protocol for the genetic transformation of Indica rice (BPT-5204). By using Agrobacterium culture with $0.5\left(\mathrm{OD}_{600 \mathrm{~nm}}\right)$ for $\mathrm{cO}^{-}$ cultivation, 2 days of co-cultivation on $100 \mu \mathrm{M}$ acetosyringone supplemented medium, high transformation efficiency $(6.2 \%)$ was achieved.

\section{Conclusion}

The result obtained in the present study gives that the optical density of the Agrobacterium, acetosyringone concentration and co-cultivation time is critical parameters that influence the transformation in indica rice (BPT-5204). The genetic transformation method developed in our study is used to developed T-DNA based activation tagging lines, which involved in develop the gain of functional mutations in recalcitrant indica rice $c v$ BPT-5204.

\section{References}

1. Aananthi N, C.R Anandakumar, R Ushakumari and P Shanthi. "Regeneration study of some indica rice cultivars followed by Agrobacterium-mediated transformation of highly regenerable cultivar Pusa Basmati." Electron. J. Plant Breed 1(2010): 1249-1256.

2. Baskaran P and I Dasgupta. "Gene delivery using micro injection of Agrobacterium to embryonic shoot apical meristem of elite indica rice cultivars." J. Plant Biochem Biotech 21 (2012): 268-274.

3. Cervera M, JA Pina, J Juarez, L Navarro and L Pena. "Agrobacterium mediated transformation of citrange factors 
affecting transformation and regeneration." Plant Cell Reports 18 (1998): 271-278

4. Dellaporta SL, J Wood, JB Hicks. "A plant DNA minipreparation Version II.” Plant MolBiol Rep 1 (1983):19-21

5. Fitch MMM, TCW Leong, Mc Cafferty, HRK, YJ Zhu, PH Moore, D Gonsalves, HS Aldwincle and HJ Atkinson "Improved transformation of Anthurium." Horticultural Science 46 (2011): 358-364.

6. Gelvin SB. "Agrobacterium and plant genes involved in TDNA transfer and integration." Annu Rev Plant Physiol Plant MolBiol.51 (2000): 223-56

7. Gelvin SB. "Agrobacterium-mediated plant transformation: the biology behind the "gene-jockeying" tool." Microbiol. Mol. Biol. Rev. 67 (2003):16-23.

8. Hiei Y, T Komari. “Agrobacterium-mediated transformation of rice using immature embryos or calli induced from mature seed.” Nat Protocols. 3 (2008): 824-834.

9. Hao ZN, J Wang, LP Wang, RX Tao. "Influences of the disease resistance conferred by the individual transgenes, Pi-d2, Pi-d3 and Xa21, on the transgenic rice plants in yield and grain quality." African J Biotech. 8 (2009):4845-4848

10. Jung KH, G An, PC Ronald. "Towards a better bowl of rice: assigning function to tens of thousands of rice genes." Nat Rev Genet 9 (2008):91-101

11. Khush GS. "Origin, dispersal, cultivation and variation of rice.” Plant MolBiol 35 (1997):25-34

12. Krishnaiah K, NRG Varma. "Changing insect pest scenario in the rice ecosystem. A national perspective." Directorate of Rice Research Rajendranagar, Hyderabad. (2012):2-8

13. Kavitha G, F Taghipour and F Huyop. "Investigation of factors in optimizing Agrobacterium-mediated gene transfer in Citrulluslanatus cv. Round Dragon." Journal on Botanical Sciences. 10(3) (2010): 209-216

14. Kumlehn J, L Serazetdinora, G Hensel, D Becker and H Loerz. "Genetic transformation of barley (Hordeum vulgare L.) via infection of andro genetic pollen culture with Agrobacterum tumefaciens." Plant Biotechnology 4 (2006): 251-258.

15. Manimaran P, G Ravikumar, M Raghurami Reddy, S Jain, TB Rao, SK Mangrauthia, RM Sundaram, S Ravichandran, SM Balachandran. "Infection of early and young callus tissues of indica rice BPT-5204 enhances regeneration and transformation efficiency.” Rice Sci 20 (2013):415-426

16. Manimaran P, S Venkata Reddy, M Moin, M Raghurami Reddy, P Yugandhar, SS Mohanraj, SM Balachandran, PB Kirti. "Activation-tagging in indicarice identifies a novel transcription factor subunit, NF-YC13 associated with salt tolerance.” Sci Rep 7 (2017):9341
17. Mourgues F, E Chevreau, C Lambert and A Bondt. "Efficient Agrobacterium mediated transformation and recovery of transgenic plants from pear (PyruscommunisL)." Plant Cell Reports.16 (1996): 245-249

18. Rance IM, W Tian, H Mathews, AD Kochko, RN Beachy, C Fauquet. "Partial desiccation of mature embryo-derived calli, a simple treatment that dramatically enhances the regeneration ability of indica rice." Plant Cell Rep.13 (1994): 647-651

19. Rashid H, S Yokoi, K Toriyama and K Hinata. "Transgenic plant production mediated by Agrobacterium in indica rice." Plant Cell Rep.15 (1996):727-730.

20. Skamnioti P, SJ Gurr. "Against the grain: safeguarding rice from rice blast disease." Trends Biotechnol 27(3) (2009): 141-150

21. Shimoda N, A Toyoda-Yamamoto, J Nagamine, S Usami, M Katayama, Y Sakagami and Y Machida. "Control of expression of Agrobacterium vir genes by synergistic actions of phenolic signal molecules and monosaccharides." Proceedings of National Academic Sciences.87 (1990): 6648-6688

22. Tripathi $\mathrm{R}, \mathrm{H}$ Bisht and $\mathrm{R}$ Singh. "Effect of Acetosyringone and Callus Age on Transformation for Scutel-lum-Derived Callus of Rice.” International Journal of Pharma and Bio Sciences. 4 (2010): 163-170

23. Upadhyaya NM, QH Zhu, RS Bhat. “Transposon insertional mutagenesis in rice. In: Pereira A (ed) Plant reverse genetics, methods and protocols, methods in molecular biology." Springer Science+Business Media. LLC (2011): 147-177

24. Urushibara S, Y Tozawa, MK Kobayashi and K Wakasa "Efficient transformation of suspension-cultured rice cells mediated by Agrobacterium tumefaciens." Breeding Sci. 51 (2001):33-38

25. Zhao Q, J Jing, G Wang, JH Wang, YY Feng, HW Xing and CF Guan. "Optimization in Agrobacterium-mediated transformation of Anthurium andraeanum using GFP as a reporter." Electronic Journal of Biotechnology.13 (2010):1-

26. Zhang Y. and T Sompong. "Optimization of Certain Parameters for Transformation of indica Rice HomKra Dang Ngah Variety via Agrobacterium-Mediated Transformation.” Kasetsart J. (Nat. Sci.). 49 (2015): 676686.

\section{Cite this article as:}

Mahendranath Gandikota, Krishnakanth Yadav T., Jagadish P., Raghurami Reddy M., Balachandran S.M., , Siddiq E.A., Yamini K.N., Anuradha G. Standardization of Agrobacterium mediated genetic transformation in Indica rice cv BPT-5204. Annals of Plant Sciences 7.2 (2018) pp. 2037-2041.

do http://dx.doi.org/10.21746/aps.2018.7.2.9

Source of support: Director, Botanical Survey of India Conflict of interest: Nil 